\title{
14
}

\section{Benchmarking for implementing a new product strategy}

\section{P.G. Pettersen}

The Norwegian Institute of Technology, Faculty of Economics and Industrial Management, N-7034 Trondheim, Norway

\section{INTRODUCTION}

Benchmarking has often been criticized for being a numerous game, comparing more or less comparable metrics between different companies. These comparisons have mostly been used to evaluate a company's performance by looking at what is possible and necessary to keep up with competitors. In many benchmarking studies, little information has been generated in respect to why there are gaps in performance between the company and other companies.

This paper is meant to give an example of how benchmarking can be used as a problem solving tool, more than merely a tool for comparison or evaluation. A case ${ }^{*}$ is presented where a company is facing problems with implementing a chosen product strategy. Implementation requires process re-engineering as well as understanding and adoption of the new strategy throughout the organization. Benchmarking is, in this case, used as a tool for identifying and analyzing different ways for implementing the chosen strategy, based on learning from other organizations in similar situations.

Definitions and theories concerning the subjects of benchmarking and product strategies will also be briefly touched in this paper.

\section{THE COMPANY AND IT'S CHALLENGE}

The case company belongs in the engineering industry. It manufactures quite complex products, which requires high skills among both engineers and operators. The company has about 400 employees, and faces international competition as $70 \%$ of the turnover comes from export. The company is recognized for it's high product quality and ability to meet customers' requirements. Although the company offers a set of more or less standard products, the customers are often technically skilled and therefore define in detail the product they want and the set of capabilities they expect from the product. This leads to an extensive need for engineering for each customer order, but also gives the customer a very customized product.

Considering the three main performance indicators (1)quality, (2) cost and (3) cycle time, the company struggle with the latter two. Product quality is satisfactory, but as in most industries, customers' expectations to cycle time and cost reduction have to be considered. The degree of customization drives both cost and cycle times, due to the extent of engineering initiated by each customer order. The extensive engineering work often leads to problems with meeting the scheduled delivery time, and it also delays the purchasing orders and

\footnotetext{
"The referred case is a part of a benchmarking project in the TOPP program, which is a government supported program for productivity improvement in Norwegian engineering industry. In the project, researchers and consultants work as facilitators for the companies, to help them implement benchmarking as a improvement tool, and to solve a problem of current interest for the companies.
} 
specifications to the company's suppliers so that purchased parts arrive too late to fit into the production and delivery plans. As a consequence, the company must either give up the delivery time, hold a considerable stock of components, or pay the suppliers more to make them shorten their delivery time to the company.

\section{A BRIEF LOOK AT THE PRODUCT STRATEGY}

It can be stated that the company is following a differentiation strategy, as it is offering higly customized and specialized products to it's customers. In a theoretical context, using Michael Porter's (1) model for categorization of product strategies, three generic product strategies are defined. These are:

- Cost leader strategy, which implies low cost products, often produced in a large scale.

- Differentiation stratcgy, which implies that the product contains attributes or features that make it different from other products. This can be made by using special brands, specific materials and capabilities etc.

- Focus strategy, which implies offering the product to a specific market segment, defined either by geographic or demographic characteristics.

In Norwegian engineering industry, the differentiation and/or the focusing strategies tend to be dominating. This can be explained by the relatively high production costs in Norway, and the fact that most of the mechanical industry delivers to the industrial market, which often requires customized products. This is also the situation for the case company.

The latest years, one has experienced a significant globalization of markets. Needs and desires converge and become homogenized all over the industrialized world, and at the same time, barriers of trade are removed. It will often be a mistake to believe that one can justify high costs (and price) by following a differentiation or focus strategy. This may be possible over a short period of time or under market conditions with high entry barriers or restrictions. However, given a strong international competition, someone will sooner or later attack your market segment, offering a better product at a lower price. A prime example of this is Mercedes Benz, who has lost a significant market share in the U.S. to Toyata's Lexus-model. At one-half the price of a Mercedes-Benz, one can have a virtually trouble-free, high performing Lexus with the same (or better) features as the Mercedes. This also shows that brand loyalty do not overcome the power of scarcity. It can therefore be argued that Porter's (1) model with the three generic strategies to a certain extent looses it's relevance for companies that compete on an international arena.

Andrews (2) states that the only long-term success strategy is to offer high value products (which means products that give the customer at least what he wants) to a low price. It is well known that mass production gives a potential for lowering costs. But from Henry Ford's mass production philosophy in the early part of the century ("everyone can have the color they want on their T-Ford - as long as it is black") and up till now, there has been a tremendous development in manufacturing systems. Today, there is a much higher degree of flexibility in the production line, and through new philosophies as for instance Just-In-Time-Production, a great variety of products can be produced in the same production line, and to a lower cost. This opens the possibility for mass production (which in general implies low costs), but also gives the customer a great variety of products to choose among. This is achieved by standardizing both parts of the product and parts of the production process.

The case company realizes that their customers must be given a high value product to a 
competitive price. Today, the customers are given a relatively high value product due to the heavy customization, but this results in high costs because of the extent of engineering work for each order. Therefore, there must be a trade-off between expensive customizing and cost reducing standardizing. The company has identified a solution to this dilemma, by distinguishing between basic parts of the product, and auxiliary parts. A strictly limited number of "basic" models are defined, and these are supplied with different combinations of auxiliaries to constitute the end product. The number of auxiliary parts being used will be limited and defined up-front, but can be combined in different ways to make the requested end product. In this way, the extent of engineering will be reduced, as well as the variety of parts or components being used in the production line. This will reduce the engineering costs, and simplify the handling of components. Still, the end product can be given a wide range of different features by combining the auxiliary parts in different ways. In this way, a great deal of the process from order to delivery can be standardized, without reducing flexibility and ability to meet customers' requirements.

However, the problem for the company is to implement this strategy, resulting in questions like: "How can this be done? What systems will be needed? What will the process look like? How will this affect the different functions in the company?" A lack of answers to these questions have been obstructing the implementation of the strategy. The main question is therefore if benchmarking can be a proper tool for helping the company find a way to implement the strategy.

\section{DEFINING BENCHMARKING}

There are lots of different definitions of benchmarking, and many of them tend to cover every aspect of benchmarking in one sentence. For all practical purposes, a simple definition of benchmarking, in business terms, can be as follows: "Benchmarking is the process of comparing something or someone with best practice."

This is a generic definition, and out of this, benchmarking can take place in several forms. Benchmarking is certainly about comparing, but can be defined in respect to what to compare and whom to compare against. Depending on what to compare, benchmarking can be divided into three main categoriess:

- Performance benchmarking.

- Process benchmarking.

- Strategic benchmarking.

Performance benchmarking is very much about comparing performance levels against "the best." The focus can be on the whole company or parts of it (e.g., functions, processes, products, departments etc.). Given that one find measures that are comparable, this can be an important contribution for motivating and convincing the organization that improvement is both possible and necessary. The problem is often that the metrics themselves do not say anything about how you can reach a better level of performance.

Process benchmarking goes one step further, as it tries to identify why someone is better. It focuses on other organizations' practices and methods that enable their high level of performance. Which companies to compare against, depends on who is perceived as the best. The best companies can be identified by doing performance benchmarking up front, and a typical benchmarking study will often be a mixture between performance and process benchmarking. 
Strategic benchmarking is more about comparing strategic corporate decisions, on for example the allocation of resources, investments, selection of business ventures, technological evolution, or development of market segments. This can be an important information to the company's own strategic planning.

In respect to whom to compare against, benchmarking can take place in one of the following three categories:

- Internal benchmarking.

- Competitive benchmarking.

- Generic (or functional) benchmarking.

Internal benchmarking is comparison between units within one organization. This method is often used by large or worldwide corporations, and is often easy to undertake, as the information is quite easy to get and is often standardized between the different units. However, the potential for real performance breakthroughs is rather small, as you seek information within your own, already well-known environment.

Competitive benchmarking implies comparison with your competitors. This could give very interesting and useful information. However, the problem is that one seldom get any useful information at all, because nobody want to share sensitive information with their competitors. Besides, there are difficult legal and ethical issues that must be considered in this type of benchmarking. Therefore, competitive benchmarking will most often either take place as a superficial comparison of key performance indicators (metrics) based on public available information, or as a comparison of metrics between a group of companies that have given their information in an anonymous way.

Generic benchmarking is comparison against companies in different industries or at least non-competitors. It is often hard to use metrics because the companies can be very different, but a more qualitative approach can be taken, and there are always some common processes or functions that can be compared. The result of benchmarking across industry borders can often be the identification and adaption of practices and methods that have been totally unknown to your own industry. Examples are the bar coding system that has spread from industry to industry, or the "invention" of the Just-In-Time concept, which can be traced back to Taichi Ohno's observation of how the supermarkets handle their throughput.

A typical benchmarking study consists of a number of steps. Watson (3) presents an useful six-step model for benchmarking, consisting of:

1) A planning step (what to benchmark, documentation, measurement)

2) A searching step (search for benchmarking partners).

3) An observing step (visit benchmarking partners).

4) An analyzing step (analyze findings, find and explain gaps).

5) An adapting step (identify what is worth adapting to your own organization).

6) An improving step (implement changes, follow up).

Whatever way benchmarking is defined and whatever model is used, it is of most importance to stress that benchmarking is about learning. The focus for benchmarking has in fact chanced over the past years from being a tool for evaluation of current performance (and trend), to be an effective way of learning methods and practices from the best. Second, it is important to understand the difference between benchmarking and so-called "industrial tourism". Effective benchmarking requires a much higher degree of planning and systematic approach in general, than a plain observation. 


\section{BENCHMARKING IN THE CASE COMPANY}

The company has been discussing how benchmarking can be used to solve some of the problems concerning cycle time and cost. Benchmarking is expected to contribute in two different, but still connected ways. A benchmarking study will be used both to analyze the critical process for the company, and to learn from others how a successful change in product strategy can be carried out.

A benchmarking team has been formed, consisting of four participants, representing the areas of marketing/sales, engineering, planning, and division management. Thus, roles as process owner, operators, internal suppliers and customers, and sponsor (management commitment) are all taken care of in the team. The team has defined the following areas as subjects to benchmarking:

1) The process from order entry to purchasing.

2) The way of changing product strategy from customized to more standardized products.

By focusing on the process from customer order to purchasing, the team is given a good understanding of the current situation, a basis for discussions with benchmarking partners and a possibility to streamline and optimize the process given it's constraints.

By focusing on the changing process, going from customized to more standardized products, it is possible to collect information concerning how this has been done in other companies, with specific focus on companies with successful experiences with such changes. The challenge is to decide the appropriate mixture between standardization and customization (given the customer requirements), to design new management systems, information systems, and technical systems that are needed to carry out the strategy, and to identify a way for implementing these systems.

The planning step in the project is crucial. According to experience from previous benchmarking studies, $50 \%$ of the total time should be used in the planning phase of the study. After choosing the area to benchmark, a lot of work must be done concerning documenting the chosen process and identifying measures. This is necessary to get knowledge of what the current situation is, and is done by going through written procedures and descriptions, and interviewing process participators. Based on relationship maps, flow-charts and verbal descriptions of the process, a discussion will take place among the team members. During this work, the team members become aware of what the entire process looks like, and where there seems to be needs and room for improvements. At the same time, the work results in a documentation that will be used later in the benchmarking study as an introductory explanation of the situation to benchmarking partners.

Compared to the definitions of benchmarking stated above, it is clear that the company wants to use benchmarking for learning. During the planning phase, the team members identify areas of improvements just by analyzing and discussing the process across the traditional borders between different functions inside the organization. In addition, by observing other successful companies that are or have been in the same situation, a lot of information and knowledge for improvements can be gained.

The type of benchmarking the company carries out, is a mixture between both strategic, process, and performance benchmarking. However, most effort is laid on process benchmarking. The first part of the benchmarking study will be focusing on the process itself, while the last part will be dealing with the enablers that make the company able to carry out the process as they would like to, and to implement the chosen strategy (i.e., the change from customization to standardization). 
When it comes to whom to compare with, the company has chosen to look at other companies with similar challenges. This will neither be companies inside their own group (internal benchmarking), nor competitors. There are no appropriate partners with the same type of challenge within their own group, and a comparison against competitors is difficult because information concerning the chosen area for benchmarking is too sensitive and strategic important to be shared. Therefore, it has been decided to carry out a generic benchmarking study. As a start, the search for benchmarking partners is done inside the company's own industry, but not among competitors. A company with similar structure and technological level will be the most appropriate for comparison. For a future study, it is also possible to look companies in totally different industries. However, because this is the company's first benchmarking study ever, the ambitions has to be held within what is realistic. It may also be important to create fast and tangible results from this first study, to use it as motivation for future studies. Anyway, the team is very open minded to the thought of looking over the fence to a complete different industry in a future study.

\section{CONCLUSION}

Benchmarking has been looked upon as merely a method for comparing and evaluating performance among organizations. Except for the fact that benchmarking implies comparing against the best or best practice instead of the average, there has not really been anything that has differed benchmarking from other methods for comparing performance measures or metrics. In the process of comparing numbers, the output is never better than the input. Consequently, if one do not know what is behind the numbers, one can not interpret or make any conclusions from the benchmarking results. Benchmarking has therefore been criticized for being a "numerous game", where "apples-to-pears"-comparisons have been made, and where the findings seldom have given information concerning why someone has been better than others.

However, the last few years, the term "process benchmarking" has gained more attention. This has lead to more focus on how other (i.e., the best) do their work, instead of just what they achieve. Thus, benchmarking has become a method for learning and problem solving, as well as a tool for evaluation.

The purpose of this paper has been to argue that benchmarking can be used in a more active way for problem solving and attacking different challenges by learning from other organizations. It has also been argued that the best pay-off from a benchmarking project occurs when focusing on process benchmarking and comparing against other industries (i.e., generic benchmarking). Although the benchmarking project, referred to as a case in this paper, has not been completed yet, it shows how benchmarking can be used as a problem solving tool rather than merely a tool for evaluating and comparing performance.

\section{LITER ATURE}

1. M. Porter, Competitive Advantage, Creating and Sustaining Superior Performance, New York, The Free Press, 1985.

2. B. Andrews, Standardizing Products and Services, Continuous Journey, No. 3 (1994) 22.

3. G. Watson, The Benchmarking Workbook, Cambridge Massachusetts; Productivity Press, 1992. 